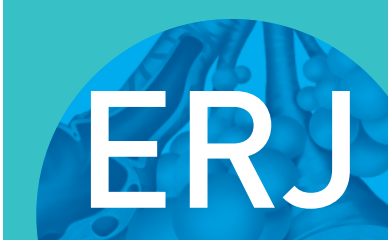

open research

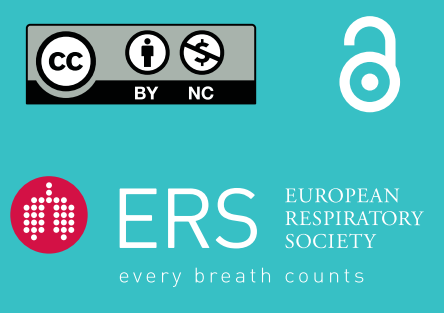

\title{
A home-based lower limb-specific resistance training programme for patients with COPD: an explorative feasibility study
}

\section{To the Editor:}

Peripheral muscle weakness leading to poor exercise tolerance is a significant problem in patients with chronic obstructive pulmonary disease (COPD). Increased lower limb strength following pulmonary rehabilitation (PR) has been shown to be associated with positive outcomes [1]. Despite this, access to and completion of PR remains problematic and alternative exercise interventions are sought. Elastic band resistance training has recently been shown to be as effective as conventional outpatient free-weight exercise training [2-4]. Elastic band resistance training may, therefore, provide an alternative home-based exercise intervention for patients with COPD who are unable or unwilling to participate in PR due to significant symptom burden or anxiety in attending group classes

The aim of this study was to investigate feasibility and acceptability of a home-based lower limb-specific resistance training exercise intervention (LLSRT) to patients with COPD. The LLSRT intervention was modelled on the strength components of PR [5-7] and strength and conditioning training literature in older adults [8].

Adults with a respiratory physician-confirmed diagnosis of COPD, exertional breathlessness (Medical Research Council (MRC) dyspnoea score $\geqslant 2$ ) and a stable smoking status, who had not completed standardised exercise in the year prior to the study were eligible. Those with a cardiac pacemaker, coexisting neurological condition, or a recent change in medication or exacerbation requiring admission (preceding 2 weeks), or those currently exercising (structured training three or more times per week within the last month) were excluded. Ethical and local research and development approval was obtained prior to commencing this research (LREC protocol number 14/LO/1817, research and development study reference KCH14-170). All participants provided written informed consent.

The LLSRT intervention was delivered and supervised by a physiotherapist (H. Cook) in the patient's own home over a 6-week period. The LLSRT was standardised and progressive (table 1), and tailored to individual patients' ability. LLSRT was supervised in the patients' homes twice weekly and participants were encouraged to complete one unsupervised session, i.e. 3 sessions per week and 18 sessions overall, each session lasting $\leqslant 45 \mathrm{~min}$ [9]. Exercise consisted of squats, sit to stand, heel raises, seated knee extensions and seated foot raises $(3 \times 10$ of each) using elastic resistance bands. Each participant was given a $\log$ book with photographic and written instruction on how to perform and progress each exercise. Progression was determined by the physiotherapist when participants were able to complete, with ease, 10 repetitions of the exercises three times per week, in addition to a reduction in their reported modified Borg breathlessness and leg fatigue scores of $\geqslant 1$. No formal education sessions were given. Feasibility outcomes included the number of patients referred and screened for eligibility, the proportion of eligible patients contacted and subsequently consented, the proportion of patients completing the 6-week LLSRT

@ERSpublications

It is feasible and acceptable to deliver a home-based lower limb-specific resistance training (LLSRT) programme to patients with COPD. Individual patient improvements in walking distance and breathlessness severity were observed post-LLSRT intervention. http://bit.ly/ 30xYpWI

Cite this article as: Cook H, Reilly CC, Rafferty GF. A home-based lower limb-specific resistance training programme for patients with COPD: an explorative feasibility study. ERJ Open Res 2019; 5: 00126-2018 [https://doi.org/10.1183/23120541.00126-2018]. 
TABLE 1 Exercise intervention: home-based lower limb-specific resistance training

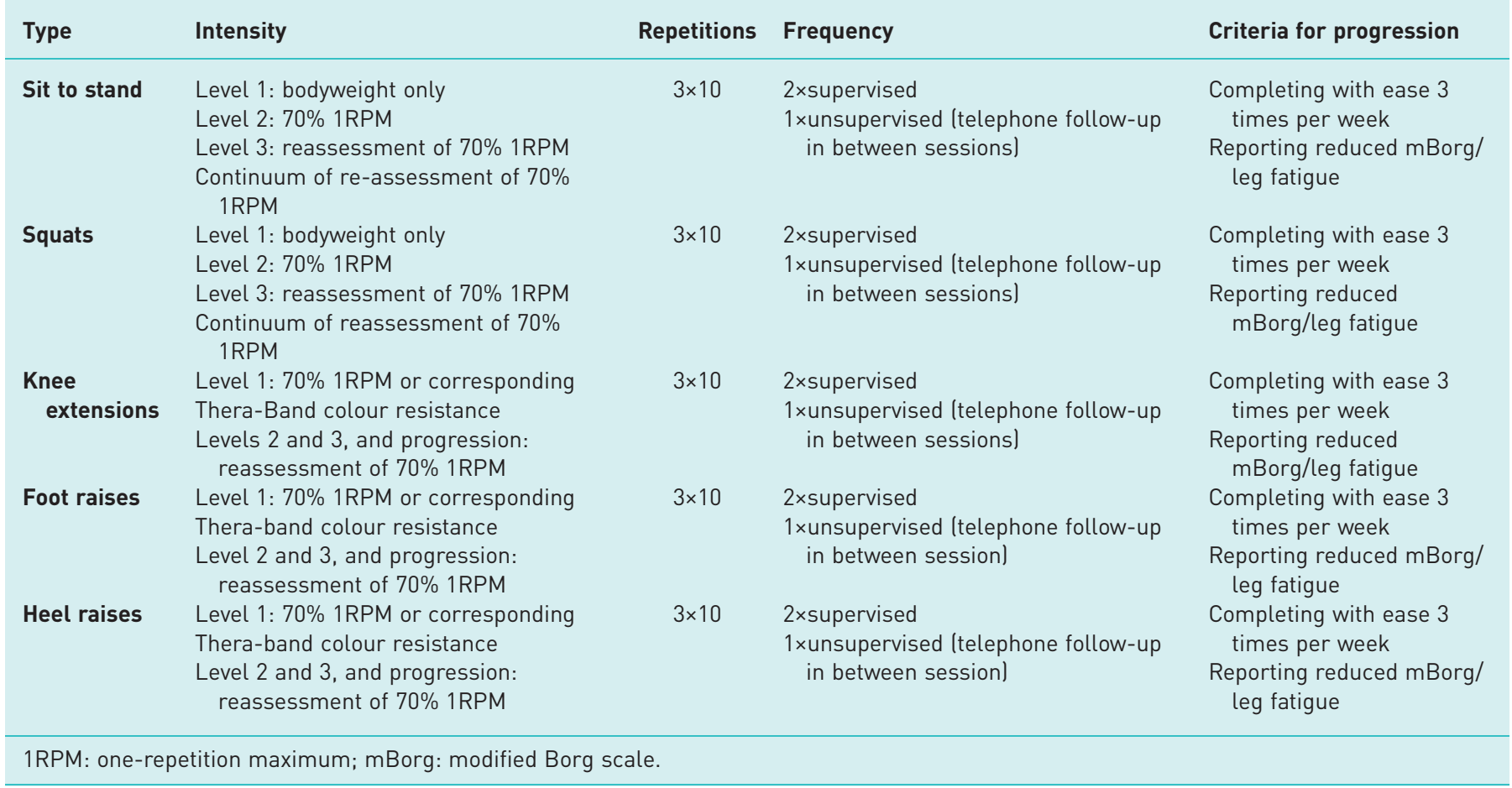

intervention, the proportion of and reasons for patients with missing data e.g. dropout rates, and compliance with the LLSRT intervention.

Pre-post-interventional clinical outcomes included exercise capacity, quadriceps strength and breathlessness severity. The incremental shuttle walk test (ISWT) was used to assess exercise capacity. A practice ISWT was performed $30 \mathrm{~min}$ prior to the walk test as per the European Respiratory Society/American Thoracic Society technical standard "Field walking tests in chronic respiratory disease" [10]. As described by SEYmour et al. [1], right quadriceps strength was assessed by maximum isometric voluntary contraction force (QMVC). Supine QMVC was measured using a quadriceps testing chair [11]. Participants were asked to complete at least five active QMVC manoeuvres during which strong verbal encouragement was given. QMVC was the maximum 1-s mean force produced. Breathlessness severity was measured across two different domains: worst at rest and worst on exertion (previous $24 \mathrm{~h}$ ) using the numerical rating scale (NRS). NRS anchors were 0 (not breathless at all) and 10 (breathlessness as bad as you can imagine).

Pre-post-interventional clinical outcomes were completed at the Respiratory Muscle Laboratory at King's College Hospital NHS Foundation Trust (London, UK). Due to the small sample size, no statistical analysis was performed; pre-post-clinical outcome data are described using descriptive statistics, and reported as median (range).

Semistructured interviews with open-ended questions were conducted to examine the perspectives of patients on the LLSRT intervention. Questions explored possible facilitators and enablers to the LLSRT intervention. Personal perspectives regarding delivering and receiving the LLSRT intervention at home were explored. Participants were asked questions regarding the impact of the LLSRT intervention on their symptoms and activities of daily living. All interviews were audio recorded and transcribed verbatim. Transcripts were analysed using deductive thematic analysis and themes were corroborated by a second researcher [12].

Over a 6-month period, 17 patients were screened for inclusion; of those, four (24\%) were ineligible, having enrolled in or recently completed PR, and two (12\%) due to medical reasons. Of those deemed appropriate for inclusion, nine (81\%) agreed to participate with seven $(66 \%)$ subsequently giving informed consent. Six patients (three male; median (range) age 66 (33-79) years, MRC dyspnoea score 3 (2-3), NRS breathlessness at rest $2.5(0-6)$ and on exertion 8.5 (7-10), forced expiratory volume in $1 \mathrm{~s}\left(\mathrm{FEV}_{1}\right) 32$ (20-78)\% predicted, and $\mathrm{FEV} 1 /$ vital capacity (VC) ratio 43 (32-79)\%) completed the intervention.

We must acknowledge that one patient (33-year-old female) had an FEV1/VC of 79\%. This patient had a clinically documented diagnosis of COPD by a respiratory physician based on: 1) a clinical history of 
10 pack-years cigarette smoking, and 10-year history of heavy cannabis smoking and recreational drug use; and 2) a computed tomography scan that confirmed emphysema and apical bullae. This patient had previously undergone a right upper lobe bullectomy (2006), which would account for the discrepancy between their spirometry and diagnosis of COPD. The patient had been previously referred to PR but had failed to attend due to a combination of social factors and anxieties around how people would "view her attendance" at PR given her age and previous cannabis use. We felt it was important to include this patient in the study as our intervention seeks to provide an alternative exercise intervention for patients with COPD who are unable or unwilling to engage with conventional PR classes.

None of the patients were receiving long-term oxygen therapy or used walking aids. Five had experienced an exacerbation in the previous year. Participants were identified via a research database aligned to our clinical PR service and therefore representative of our local patient population. The patients were symptomatically breathless, had clinically documented anxieties or apprehensions regarding attending group exercise resulting in nonattendance of $\mathrm{PR}$, or had previously attended and not maintained exercise independently, indicating a local need for an alternative exercise intervention. This pattern is in keeping with the national PR audit (England and Wales 2017), which indicated that 33\% of patients referred to PR fail to attend their initial assessment [13].

Adherence to the two supervised sessions and the recommended weekly self-guided, unsupervised exercise session was $100 \%$ for all patients. A median (range) improvement of $50(-10-170) \mathrm{m}$ was observed post-LLRT for the group. On an individual-patient level, incremental shuttle walk distance (SWD) increased in five of the six participants, of whom three improved by more than the minimal clinically important difference (MCID) of $47.5 \mathrm{~m}$.

Post-LLRST, QMVC increased by 3.57 (-1.5-14) kg; QMVC increased in three participants, remained unchanged in two and decreased in one participant at 6 weeks. NRS breathlessness scores were reduced by $-1.5(0--4)$ at rest and $-2.5(-1--7)$ on exertion post-LLRT. Breathlessness was reduced in five of the six participants; improvements in NRS were greater than the MCID.

Two themes emerged from the qualitative interviews: "acceptability of the exercise intervention"; and "value of the intervention", where patients found the LLSRT intervention acceptable, worthwhile and a beneficial experience.

All patients felt the 6-week exercise intervention was acceptable, valuable and of benefit them.

It's a worthwhile thing [LLRT] to have done.

Participant 6, line 63

I could feel those [leg] muscles being used and I was really surprised [...] Certainly mine [leg muscles] are a lot better now; the penny drops and you think, crikey! This is brilliant.

Participant 5, line 94-95

Participants acknowledged the support they received throughout the intervention and reported that the frequency of the supervised session was acceptable.

Twice a week is enough; should not be more or less. The supervision from this programme improves confidence as a physiotherapist is guiding you at home.

Participant 1 , line $16-18$

Support was provided not only through face-to-face supervision but also via telephone support as required, and this was perceived as a positive aspect of the intervention. Furthermore, the provision of the exercise log to assist and support the unsupervised sessions was deemed acceptable and helpful.

Interviewer: And you felt confident enough to do the exercises by yourself?

Patient: Yes, the physiotherapist explained them [...] and the literature they gave us [the exercise $\log$ ] and the visuals on it, were very useful, which I thought was very good to have.

Participant 5, line 27-28

All six patients reported that they valued and would recommend this intervention to other patients with COPD.

Found sit to stand enjoyable [...] Can't think that the exercise programme could be improved anymore though; just the right amount of time. I would definitely recommend. 
The personalised approach to this home-based programme of exercises was appreciated by all patients. It was described by patients as a "good way" of starting to exercise again and was perceived by patients as "prehab" or "a good introduction" to exercise.

Patients described a sense of positivity towards the intervention and felt that it had allowed them to improve their physical strength/ability, which translated into being able to do more at home.

I'm doing more at home. I went for an appointment, came back and I did the gardening, mowed the lawn, and I'm thinking, how come? I wouldn't have done that normally; it would have been too much to manage. Usually, one big activity a day is enough for me. I've been taking on much more and I think, ah! This is good.

Participant 5, line 55-56

I am doing a little bit more; I can do the washing and scrubbing down of the tiles and the sink, pottering around doing bits and pieces.

Participant 4, line 31

My walking has improved, and finding it easier to walk up the stairs and do activities at home.

Participant 1 , line 10

Activities of daily living improved and symptomatically, patients felt better.

Both my wife and I think things have improved; breathing better, controlling my breathing more and not so afraid.

Participant 2, line 2-3

Patients reported improved confidence and coping abilities after the intervention.

Sometimes it's my confidence with my breathing which more than anything affects me. Sometimes I think, oh I can't go out today [...] This has got a bit better.

Participant 4, line 37-43

Being informed of their improvements/progression whilst undertaking the intervention consolidated confidence improvements.

I really enjoyed it and looked forward to the days the physiotherapist was coming to the house. Seeing my results has made me more confident.

Participant 2, line 2-3

One participant reported they were coping better, which came directly from symptom improvement.

I feel my breathing has got a lot better [....] I certainly feel I cope better, whether that's because when I walk, I have more strength in my legs now.

Participant 5, line 39-40

Patients reported that they were more confident and empowered to continue to exercise after the study (positive behaviour change).

I do feel it's done me so much good; I don't want to stop it so I can keep building up on it. I feel I am coping, managing it. I think the word is "managing" a lot better now.

Participant 5, line 41-42

All participants valued the intervention being in their own homes, which increased ease in completing the exercises and did not impact on their daily activities.

It was really useful, as it saved the journey going back, which can be a whole morning for me. It didn't interrupt the flow of the day; I could still do the things I'd normally do.

Participant 3, line 17-18

Easy to complete the exercises and I didn't have to travel to attend a class.

Participant 1 , line 15

Participants who previously were not comfortable in a group environment valued this intervention as it allowed them to exercise in a "safe and familiar" space.

I don't get embarrassed in front of the physiotherapist, but when I go to the gym, I do get a bit embarrassed. 
The study demonstrated feasibility of delivering a home-based LLSRT intervention and despite the low numbers recruited, $100 \%$ compliance was achieved with regards to the supervised and unsupervised components of the intervention. The cohort of previously inactive COPD participants had started to increase activity levels by the end of the relatively short 6-week intervention. The numbers of completed treatment sessions (both supervised and unsupervised) are comparable, if not better, than some reported PR compliance and adherence data [14-16], which further supports the development of LLRT as a potential exercise intervention for patients with COPD.

Improvements in confidence to manage symptoms and complete activities of daily living increased the acceptability of the intervention. The evidence suggests low self-efficacy in COPD is linked to the spiral of inactivity [17]. As part of this short intervention, participants had motivated themselves to start exercising independently. This demonstrates that these participants found this intervention useful. This home-based exercise intervention shows promise for improving patients' ability to cope with their disease and confidence improving their own functional abilities.

Two methods of assessing breathlessness were employed, the MRC dyspnoea score and NRS, as each measures different characteristics/components of chronic breathlessness. The MRC measures the impact of breathlessness on physical function, while the NRS measures the intensity of breathlessness. In this study, we used that MRC dyspnoea score to characterise our patients and the NRS as our outcome measure. An advantage of the NRS is that it can quantify changes in breathlessness at rest and on exertion. Patients reported improvements in breathlessness intensity, which is in keeping with the PR literature $[5,6]$. Specifically training the lower-limb muscles leads to increased cardiorespiratory and muscular efficiency, therefore reducing the load on the respiratory muscle pump, most notably during exercise, resulting in decreased breathlessness intensity. To further explore this observation, future studies would benefit from the inclusion of both volitional and nonvolitional measures of lower-limb strength [11] as well cardiopulmonary exercise testing and measurement of oxygen uptake at the anaerobic threshold.

Functionally, all apart from one patient improved in the ISWT, and three by the MCID (49.5 m). All participants stopped due to ventilatory limitation rather than leg fatigue. Given the symptomatic nature of this cohort, future studies may benefit from the addition of the 6-min walking test, which may corroborate the qualitative perceived improvements in function. In addition, the five-repetition sit to stand or 1-min sit to stand test, or the short physical performance battery [18], could provide an alternative to the ISWT, with the added advantage of being performed in the patients home, reducing sample size and participant study burden. The study included patients with a broad range of ages and lung function. Despite the youngest patient showing the greatest improvement in ISWT, the older participants with worse lung function also showed improvements greater than the MCID for ISWT and showed the greatest improvement in quadriceps strength. Age or lung function at baseline did appear to influence the outcome of this intervention.

The lack of an observed improvement in the QMVC may be attributed to the nature of the QMVC test and the impact of patient cooperation and motivation. In addition to a control group, the use of nonvolitional measures of quadriceps function would provide a more robust assessment of any changes in in strength and its impact on with the functional and symptomatic improvements observed in this study.

We acknowledge that both aerobic and resistance exercise training are recommended for patients with COPD; however, we developed this intervention based on the findings of SEYMOUR et al. [1], which demonstrated that following completion of $\mathrm{PR}$, patients increased their quadriceps strength, resulting in improved ISWD and health-related quality of life. In addition, SEYmour et al. [1] reported that a fall in quadriceps strength was more likely if a re-exacerbation event occurred (OR 3.87, 95\% CI 1.01-12.2; $\mathrm{p}=0.049$ ), demonstrating the importance of lower-limb muscle strength in patients with COPD. Moreover, in patients with advanced COPD (MRC dyspnoea score 4-5), who are unable to engage with PR, specifically targeting the quadriceps muscle using neuromuscular electrical stimulation over a 6-week period, increased functional exercise capacity by enhancing quadriceps muscle mass and function [19]. Such studies provided the pathophysiological basis for our LLRT intervention.

The lead researcher who supervised the exercise sessions also conducted the post-interventional, semistructured, qualitative interviews. To account for any bias associated with this approach, transcripts and analysis were reviewed independently by a second researcher (C.C. Reilly), and final themes agreed via consensus.

An intervention period of $>6$ weeks may have resulted greater improvement in strength. The duration of the intervention was based on guidelines for standard PR and training in the elderly, which recommend rehabilitation programmes should be between 6 and 12 weeks with twice weekly supervised sessions [7]. Thera-band was chosen for implementing this intervention due it being a relatively inexpensive, easily 
transportable adjunct to training that has been shown to be effective in improving volitional measures of strength $[2-4,20]$. What remains to be defined is the optimal duration of this intervention and whether there is a celling effect. Cost implications of the LLSRT training programme need to be modelled and tested. Larger, adequately powered studies are required to assess the effectiveness of this intervention on physical function and health-related quality of life.

\section{Hannah Cook ${ }^{1,3}$, Charles C. Reilly ${ }^{2,3}$ and Gerrard F. Rafferty $\oplus^{1}$}

${ }^{1}$ King's College London, Centre for Human and Aerospace Physiological Sciences, London, UK. ${ }^{2}$ Dept of Physiotherapy, Kings College Hospital NHS Foundation Trust, London, UK. ${ }^{3}$ These authors contributed equally to this work.

Correspondence: Charles C. Reilly, King's College Hospital NHS Foundation Trust, Dept of Physiotherapy, 4th Floor, Hambleden Wing East, Denmark Hill, London, SE5 9RS, UK. E-mail: charles.c.reilly@kcl.ac.uk

Received: Aug 032018 | Accepted after revision: May 012019

Conflict of interest: None declared.

Support statement: This project was completed as part of a National Institute for Health Research-funded Masters in research. Funding information for this article has been deposited with the Crossref Funder Registry.

\section{References}

1 Seymour JM, Moore L, Jolley CJ, et al. Outpatient pulmonary rehabilitation following acute exacerbations of COPD. Thorax 2010; 65: 423-428.

2 Ramos EM, de Toledo-Arruda AC, Fosco LC, et al. The effects of elastic tubing-based resistance training compared with conventional resistance training in patients with moderate chronic obstructive pulmonary disease: a randomized clinical trial. Clin Rehabil 2014; 28: 1096-1106.

3 O'Shea SD, Taylor NF, Paratz JD. A predominantly home-based progressive resistance exercise program increases knee extensor strength in the short-term in people with chronic obstructive pulmonary disease: a randomised controlled trial. Aust J Physiother 2007; 53: 229-237.

4 Nyberg A, Lindström B, Rickenlund A, et al. Low-load/high-repetition elastic band resistance training in patients with COPD: a randomized, controlled, multicenter trial. Clin Respir J 2015; 9: 278-288.

5 Spruit MA, Singh SJ, Garvey C, et al. An official American Thoracic Society/European Respiratory Society statement: key concepts and advances in pulmonary rehabilitation. Am J Respir Crit Care Med 2013; 188: e13-e64.

6 Maltais F, Decramer M, Casaburi R, et al. An official American Thoracic Society/European Respiratory Society statement: update on limb muscle dysfunction in chronic obstructive pulmonary disease. Am J Respir Crit Care Med 2014; 189: e15-e62.

7 Bolton CE, Bevan-Smith EF, Blakey JD, et al. British Thoracic Society guideline on pulmonary rehabilitation in adults. Thorax 2013; 68: Suppl. 2, ii1-ii30.

8 Straight $\mathrm{CR}$, Lindheimer JB, Brady AO, et al. Effects of resistance training on lower-extremity muscle power in middle-aged and older adults: a systematic review and meta-analysis of randomized controlled trials. Sports Med 2016; 46: 353-364.

9 American College of Sports Medicine. American College of Sports Medicine position stand. Progression models in resistance training for healthy adults. Med Sci Sports Exerc 2009; 41: 687-708.

10 Holland AE, Spruit MA, Troosters T. An official European Respiratory Society/American Thoracic Society technical standard: field walking tests in chronic respiratory disease. Eur Respir 2014; 44: 1428-1446.

11 Man WD, Soliman MG, Nikoletou D, et al. Non-volitional assessment of skeletal muscle strength in patients with chronic obstructive pulmonary disease. Thorax 2003; 58: 665-669.

12 Gale, NK, Heath G, Cameron E, et al. Using the framework method for the analysis of qualitative data in multi-disciplinary health research. BMC Med Res Methodol 2013; 13: 117.

13 Steiner M, McMillan V, Lowe D, et al. Pulmonary rehabilitation: an exercise in improvement. National Chronic Obstructive Pulmonary Disease (COPD) Audit Programme: Clinical and organisational audits of pulmonary rehabilitation services in England and Wales 2017. National report. London, Royal College of Physicians, 2018.

14 Jones SE, Green SA, Clark AL, et al. Pulmonary rehabilitation following hospitalisation for acute exacerbation of COPD: referrals, uptake and adherence. Thorax 2014; 69: 181-182.

15 National COPD Audit Programme. Pulmonary Rehabilitation: Steps to Breathe Better. London, Royal College of Physicians, 2016.

16 Jones AW, Taylor A, Gowler H, et al. Systematic review of interventions to improve patient uptake and completion of pulmonary rehabilitation in COPD. ERJ Open Res 2017; 3: 00089-2016.

17 Wigal JK, Creer TL, Kotses H. The COPD Self-Efficacy Scale. Chest 1991; 99: 1193-1196.

18 Larsson P, Borge CR, Nygren-Bonnier M, et al. An evaluation of the short physical performance battery following pulmonary rehabilitation in patients with chronic obstructive pulmonary disease. BMC Res Notes 2018; 4: I.

19 Maddocks, M, Nolan CM, Man WD, et al. Neuromuscular electrical stimulation to improve exercise capacity in patients with severe COPD: a randomised double-blind, placebo-controlled trial. Lancet Respir Med 2016 4: $27-36$.

20 Kongsgaard M, Backer V, Jørgensen $\mathrm{K}$, et al. Heavy resistance training increases muscle size, strength and physical function in elderly male COPD-patients - a pilot study. Respir Med 2004; 98: 1000-1007. 